УДК 519.652

Дата подачи статьи: 17.11 .17

DOI: $10.15827 / 0236-235 X .122 .382-386$ 2018. Т. 31. № 2. C. 382-386

\title{
РАСЧЕТ КОНСТРУКЦИЙ МЕТОДОМ КОНЕЧНЫХ ЭАЕМЕНТОВ В СРЕДЕ МАТЕМАТИЧЕСКОГО ПАКЕТА MathCAD
}

\author{
C.B. Бакушев 1, д.m.н., npoфpeccop, bakuchsv@mail.ru \\ 1 Пензенский государственный университет архитектуры и строительства, \\ ул. Г. Титова, 28, г. Пенза, 440028, Россия
}

Рассматриваются возможности математического пакета MathCAD при решении задач прочностного и деформационного расчета строительных и машиностроительных конструкций методом конечных элементов. Показано, что для решения данных задач возможностей математического пакета MathCAD явно недостаточно, так как необходимо обрабатывать достаточно большие объемы информации.

Для задач иллюстративного или учебного характера, когда матрица жесткости имеет небольшую размерность и ее компоненты можно ввести в ручном режиме, возможностей пакета MathCAD вполне достаточно, и с его помощью можно получить решение задачи. При этом матрицу жесткости следует формировать путем ручного ввода значений ее компонент, вычисленных заранее в системе MathCAD. B реальных расчетных задачах матрица жесткости имеет большой порядок (порядка тысяч, десятков и сотен тысяч), поэтому для их решения следует использовать специализированные программные комплексы, например ЛИРА, SCAD, ANSYS и другие.

В статье рассмотрен конкретный числовой пример расчета пластинки сложного очертания, находящейся в условиях плоского напряженного состояния, методом конечных элементов. Пластинка разбивается на три конечных элемента. Матрица жесткости при этом имеет порядок, равный десяти, и решение задачи получено лишь при ручном вводе значений ее компонент.

Полученные результаты можно применять при решении задач расчета строительных и машиностроительных конструкций методом конечных элементов с использованием программно-математического обеспечения персональных компьютеров, в частности, MathCAD.

Ключевые слова: метод конечных элементов, матрица жесткости, математический пакет МаthCAD, персональные компьютеры.

Математический пакет MathCAD, входящий в прикладное программно-математическое обеспечение современных персональных компьютеров, является одним из наиболее популярных средств, предназначенных для решения как чисто математических, так и инженерных задач. Достоинства пакета MathCAD общеизвестны и описаны во многих печатных изданиях, в частности в [1-3]. Простота, доступность, интуитивность пользовательского интерфейса MathCAD приводят к соблазну использовать его для решения достаточно сложных инженерных задач. Действительно, в работах [4-6] и многих других решение математических и инженерных задач реализовано на базе этого математического пакета. Вместе с тем необходимо отметить, что реализация численных методов решения задач, в частности, задач механики деформируемого твердого тела, в системе MathCAD связана с определенными трудностями. Это обусловлено необходимостью обработки достаточно больших объемов информации, с которыми MathCAD зачастую не справляется. К такого типа задачам относятся и задачи расчета строительных и машиностроительных конструкций методом конечных элементов.

Это численный метод решения краевых задач для дифференциальных уравнений, в основе которого лежит разбиение области решения на конечные элементы произвольных размеров, но, как правило, одной формы [7-9]. Искомая функция (например, функция перемещений или напряжений) внутри конечного элемента аппроксимиру- ется, как правило, полиномом, коэффициенты которого выражаются через значения искомой функции в вершинах конечных элементов. В итоге решение задачи сводится к решению системы линейных алгебраических уравнений относительно значений искомой функции в узлах - вершинах совокупности конечных элементов, покрывающих расчетную область. При этом матрица жесткости, симметричная относительно главной диагонали, имеет, как правило, высокий порядок, хотя и является разреженной. Компоненты матрицы жесткости вычисляются исходя из геометрии рассматриваемой области, способа ее деления на отдельные конечные элементы, а также механических характеристик материала рассчитываемой конструкции. При вычислении перемещений в вершинах конечных элементов матрицу жесткости необходимо обратить. И вот здесь возникают проблемы.

Рассмотрим плоское упругое тело, модуль упругости которого $E=2,1 \cdot 10^{11}$ Па, коэффициент Пуассона $\mu=0,3$. Тело находится в условиях обобщенного плоского напряженного состояния. Разделим тело на три треугольных конечных элемента. Проиллюстрируем это рисунком 1, на котором арабскими цифрами обозначены номера вершин конечных элементов, римскими - номера конечных элементов, дугой со стрелкой - направление обхода вершин конечных элементов внутри каждого конечного элемента. К вершинам совокупности конечных элементов приложены составляющие внешних сил $\Phi_{x i}, \Phi_{y i}$. 


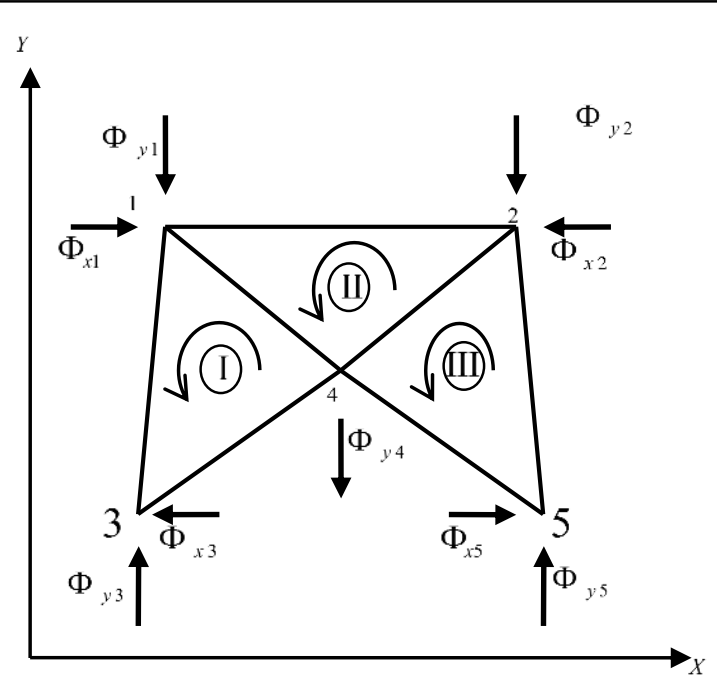

Рис. 1. Плоское упругое тело, разбитое на конечные элементы

Fig. 1. A plane elastic body divided into finite elements

Координаты вершин конечных элементов и величины внешних сил, приложенных к вершинам конечных элементов:

$$
\begin{aligned}
& x_{1}=1 \mathrm{~m}, \quad y_{1}=2,7 \mathrm{~m}, \\
& x_{2}=3,6 \mathrm{~m}, \quad y_{2}=2,7 \mathrm{~m}, \\
& x_{3}=0,8 \mathrm{M}, \quad y_{3}=0,9 \mathrm{M}, \\
& x_{4}=2,3 \mathrm{M}, \quad y_{4}=1,8 \mathrm{M}, \\
& x_{5}=3,8 \mathrm{M}, \quad y_{5}=0,9 \mathrm{~m} . \\
& \Phi_{x 1}=10^{4} \mathrm{H}, \quad \Phi_{y 1}=2 \cdot 10^{4} \mathrm{H}, \\
& \Phi_{x 2}=10^{4} \mathrm{H}, \quad \Phi_{y 2}=2 \cdot 10^{4} \mathrm{H}, \\
& \Phi_{x 3}=2 \cdot 10^{4} \mathrm{H}, \quad \Phi_{y 3}=3 \cdot 10^{4} \mathrm{H}, \\
& \Phi_{x 4}=0,0 \mathrm{H}, \quad \Phi_{y 4}=2 \cdot 10^{4} \mathrm{H}, \\
& \Phi_{x 5}=2 \cdot 10^{4} \mathrm{H}, \quad \Phi_{y 5}=3 \cdot 10^{4} \mathrm{H} .
\end{aligned}
$$

Условия равновесия упругого тела выполняются:

$$
\begin{aligned}
\sum X=0: & \Phi_{x 1}-\Phi_{x 2}-\Phi_{x 3}+\Phi_{x 5}= \\
& =(1-1-2+2) \cdot 10^{4} \equiv 0 ; \\
\sum Y=0: \quad & -\Phi_{y 1}-\Phi_{y 2}+\Phi_{y 3}-\Phi_{y 4}+\Phi_{y 5}= \\
& =(-2-2+3-2+3) \cdot 10^{4} \equiv 0 ; \\
\sum M_{4}=0: & \Phi_{x 1}\left(y_{1}-y_{4}\right)-\Phi_{x 2}\left(y_{2}-y_{4}\right)+ \\
& +\Phi_{x 3}\left(y_{4}-y_{3}\right)-\Phi_{x 5}\left(y_{4}-y_{5}\right)- \\
& -\Phi_{y 1}\left(x_{4}-x_{1}\right)+\Phi_{y 2}\left(x_{2}-x_{4}\right)+ \\
& +\Phi_{y 3}\left(x_{4}-x_{3}\right)-\Phi_{y 5}\left(x_{5}-x_{4}\right)= \\
= & {[1 \cdot(2,7-1,8)-1 \cdot(2,7-1,8)+} \\
+ & 2 \cdot(1,8-0,9)-2 \cdot(1,8-0,9)- \\
- & 2 \cdot(2,3-1)+2 \cdot(3,6-2,3)+ \\
+ & 3 \cdot(2,3-0,8)-3 \cdot(3,8-2,3)] \cdot 10^{4} \equiv 0 .
\end{aligned}
$$

Тело находится в условиях обобщенного плоского напряженного состояния, поэтому

$$
\begin{aligned}
& A=\frac{E}{1-\mu^{2}}=\frac{2,1 \cdot 10^{11}(\Pi \mathrm{a})}{1-0,3^{2}}=2,308 \cdot 10^{11} \Pi \mathrm{\Pi a} \\
& B=\frac{E}{2(1-\mu)}=\frac{2,1 \cdot 10^{11}(\Pi \mathrm{a})}{2(1-0,3)}=1,500 \cdot 10^{11} \Pi \mathrm{a} ; \\
& C=\frac{E \cdot \mu}{1-\mu^{2}}=\frac{2,1 \cdot 10^{11}(\Pi \mathrm{a}) \cdot 0,3}{1-0,3^{2}}=6,923 \cdot 10^{10} \Pi \mathrm{a} ; \\
& G=\frac{E}{2(1+\mu)}=\frac{2,1 \cdot 10^{11}(\Pi \mathrm{a})}{2(1+0,3)}=8,077 \cdot 10^{10} \Pi \mathrm{a} .
\end{aligned}
$$

Используя пакет MathCAD, вычисляем компоненты матриц жесткости конечных элементов:

$$
\begin{aligned}
& \text { КЭ }-\mathrm{I}: i \rightarrow 1, j \rightarrow 3, k \rightarrow 4 . \\
& a_{11}=A\left(y_{j}-y_{k}\right)^{2}+G\left(x_{j}-x_{k}\right)^{2}=3.687 \cdot 10^{11} \mathrm{H} ; \\
& a_{12}=-A\left(y_{i}-y_{k}\right)\left(y_{j}-y_{k}\right)- \\
& -G\left(x_{i}-x_{k}\right)\left(x_{j}-x_{k}\right)=2,942 \cdot 10^{10} \mathrm{H} ; \\
& a_{13}=A\left(y_{i}-y_{j}\right)\left(y_{j}-y_{k}\right)+ \\
& +G\left(x_{i}-x_{j}\right)\left(x_{j}-x_{k}\right)=-3,981 \cdot 10^{11} \mathrm{H} ; \\
& \ldots \\
& a_{56}=-A\left(x_{i}-x_{j}\right)\left(x_{i}-x_{k}\right)- \\
& -G\left(y_{i}-y_{j}\right)\left(y_{i}-y_{k}\right)=-7,085 \cdot 10^{11} \mathrm{H} ; \\
& a_{66}=A\left(x_{i}-x_{j}\right)^{2}+G\left(y_{i}-y_{j}\right)^{2}=2,709 \cdot 10^{11} \mathrm{H} .
\end{aligned}
$$

КЭ - II: $i \rightarrow 1, j \rightarrow 4, k \rightarrow 2$.

$b_{11}=A\left(y_{j}-y_{k}\right)^{2}+G\left(x_{j}-x_{k}\right)^{2}=3,234 \cdot 10^{11} \mathrm{H}$;

$b_{12}=-A\left(y_{i}-y_{k}\right)\left(y_{j}-y_{k}\right)-$

$-G\left(x_{i}-x_{k}\right)\left(x_{j}-x_{k}\right)=-2,730 \cdot 10^{11} \mathrm{H}$;

$b_{13}=A\left(y_{i}-y_{j}\right)\left(y_{j}-y_{k}\right)+$

$+G\left(x_{i}-x_{j}\right)\left(x_{j}-x_{k}\right)=-5,420 \cdot 10^{10} \mathrm{H}$;

$\cdots$

$b_{56}=-A\left(x_{i}-x_{j}\right)\left(x_{i}-x_{k}\right)-$

$-G\left(y_{i}-y_{j}\right)\left(y_{i}-y_{k}\right)=-7,800 \cdot 10^{11} \mathrm{H}$;

$b_{66}=A\left(x_{i}-x_{j}\right)^{2}+G\left(y_{i}-y_{j}\right)^{2}=4,554 \cdot 10^{11} \mathrm{H}$.

KЭ - III: $i \rightarrow 2, j \rightarrow 4, k \rightarrow 5$.

$c_{11}=A\left(y_{j}-y_{k}\right)^{2}+G\left(x_{j}-x_{k}\right)^{2}=3,687 \cdot 10^{11} \mathrm{H}$;

$c_{12}=-A\left(y_{i}-y_{k}\right)\left(y_{j}-y_{k}\right)-$

$-G\left(x_{i}-x_{k}\right)\left(x_{j}-x_{k}\right)=-3,981 \cdot 10^{11} \mathrm{H}$;

$\cdots$

$c_{56}=-A\left(x_{i}-x_{j}\right)\left(x_{i}-x_{k}\right)-$

$-G\left(y_{i}-y_{j}\right)\left(y_{i}-y_{k}\right)=-7,085 \cdot 10^{10} \mathrm{H}$;

$c_{66}=A\left(x_{i}-x_{j}\right)^{2}+G\left(y_{i}-y_{j}\right)^{2}=4,554 \cdot 10^{11} \mathrm{H}$. 
Вычисляем компоненты матрицы жесткости К совокупности конечных элементов (в нашем случае матрица жесткости будет десятого порядка). Расчетные формулы будут следующими:

$$
\begin{aligned}
& K_{11}=a_{11}+b_{11}=6,921 \cdot 10^{11} \mathrm{H} ; \\
& K_{12}=b_{13}=-5,042 \cdot 10^{10} \mathrm{H} ; \\
& K_{13}=a_{12}=2,942 \cdot 10^{10} \mathrm{H} ; \\
& K_{14}=a_{13}+b_{12}=-6,711 \cdot 10^{11} \mathrm{H} ; \\
& \cdots \\
& K_{8,10}=0 ; \\
& K_{99}=a_{66}+b_{55}+c_{55}=2,102 \cdot 10^{12} \mathrm{H} ; \\
& K_{9,10}=c_{56}=-7,085 \cdot 10^{10} \mathrm{H} ; \\
& K_{10,10}=c_{66}=4,554 \cdot 10^{11} \mathrm{H} .
\end{aligned}
$$

Формируем матрицу жесткости K:

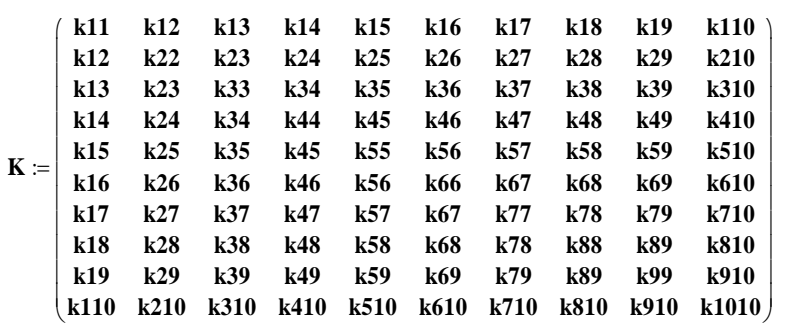

\section{Матрица жесткости К, сформированная в автоматизированном режиме}

Таблица 1

\section{An automated rigidity matrix $K$}

Table 1

\begin{tabular}{|l|r|r|r|r|r|r|r|r|r|r|}
\hline & \multicolumn{1}{|c|}{0} & \multicolumn{1}{c|}{1} & \multicolumn{1}{c|}{2} & \multicolumn{1}{c|}{3} & \multicolumn{1}{c|}{4} & \multicolumn{1}{c|}{5} & \multicolumn{1}{c|}{6} & \multicolumn{1}{c|}{7} & \multicolumn{1}{c|}{8} & 9 \\
\hline 0 & $6.921 \cdot 10^{11}$ & $-5.042 \cdot 10^{10}$ & $2.942 \cdot 10^{10}$ & $-6.711 \cdot 10^{11}$ & 0 & $-3.780 \cdot 10^{11}$ & $1.350 \cdot 10^{10}$ & $-2.804 \cdot 10^{10}$ & $3.925 \cdot 10^{11}$ & 0 \\
\hline 1 & $-5.042 \cdot 10^{10}$ & $6.921 \cdot 10^{11}$ & 0 & $-6.711 \cdot 10^{11}$ & $2.942 \cdot 10^{10}$ & $-1.350 \cdot 10^{10}$ & $1.485 \cdot 10^{11}$ & 0 & $-3.925 \cdot 10^{11}$ & $2.804 \cdot 10^{10}$ \\
\hline 2 & $2.942 \cdot 10^{10}$ & 0 & $3.234 \cdot 10^{11}$ & $-3.528 \cdot 10^{11}$ & 0 & $1.038 \cdot 10^{9}$ & 0 & $1.755 \cdot 10^{11}$ & $-1.765 \cdot 10^{11}$ & 0 \\
\hline 3 & $-6.711 \cdot 10^{11}$ & $-6.711 \cdot 10^{11}$ & $-3.528 \cdot 10^{11}$ & $2.048 \cdot 10^{12}$ & $-3.528 \cdot 10^{11}$ & $3.905 \cdot 10^{11}$ & $-3.905 \cdot 10^{11}$ & $-1.475 \cdot 10^{11}$ & $-5.341 \cdot 10^{-5}$ & $1.475 \cdot 10^{11}$ \\
\hline 4 & 0 & $2.942 \cdot 10^{10}$ & 0 & $-3.528 \cdot 10^{11}$ & $3.234 \cdot 10^{11}$ & 0 & $-1.038 \cdot 10^{9}$ & 0 & $1.765 \cdot 10^{11}$ & $-1.755 \cdot 10^{11}$ \\
\hline 5 & $-3.780 \cdot 10^{11}$ & $-1.350 \cdot 10^{10}$ & $1.038 \cdot 10^{9}$ & $3.905 \cdot 10^{11}$ & 0 & $1.040 \cdot 10^{12}$ & $3.246 \cdot 10^{11}$ & $-3.846 \cdot 10^{11}$ & $-9.801 \cdot 10^{11}$ & 0 \\
\hline 6 & $1.350 \cdot 10^{10}$ & $1.485 \cdot 10^{11}$ & 0 & $-3.905 \cdot 10^{11}$ & $-1.038 \cdot 10^{9}$ & $3.246 \cdot 10^{11}$ & $1.040 \cdot 10^{12}$ & 0 & $-9.801 \cdot 10^{11}$ & $-3.846 \cdot 10^{11}$ \\
\hline 7 & $-2.804 \cdot 10^{10}$ & 0 & $1.755 \cdot 10^{11}$ & $-1.475 \cdot 10^{11}$ & 0 & $-3.846 \cdot 10^{11}$ & 0 & $4.554 \cdot 10^{11}$ & $-7.085 \cdot 10^{10}$ & 0 \\
\hline 8 & $3.925 \cdot 10^{11}$ & $-3.925 \cdot 10^{11}$ & $-1.765 \cdot 10^{11}$ & $-5.341 \cdot 10^{-5}$ & $1.765 \cdot 10^{11}$ & $-9.801 \cdot 10^{11}$ & $-9.801 \cdot 10^{11}$ & $-7.085 \cdot 10^{10}$ & $2.102 \cdot 10^{12}$ & $-7.085 \cdot 10^{10}$ \\
\hline 9 & 0 & $2.804 \cdot 10^{10}$ & 0 & $1.475 \cdot 10^{11}$ & $1.755 \cdot 10^{11}$ & 0 & $-3.846 \cdot 10^{11}$ & 0 & $-7.085 \cdot 10^{10}$ & $4.554 \cdot 10^{11}$ \\
\hline
\end{tabular}

Единичная матрица, сгенерированная на основе матрицы жесткости (табл. 1)

Таблица 2

An identity matrix based on the rigidity matrix (Tab. 1)

Table 2

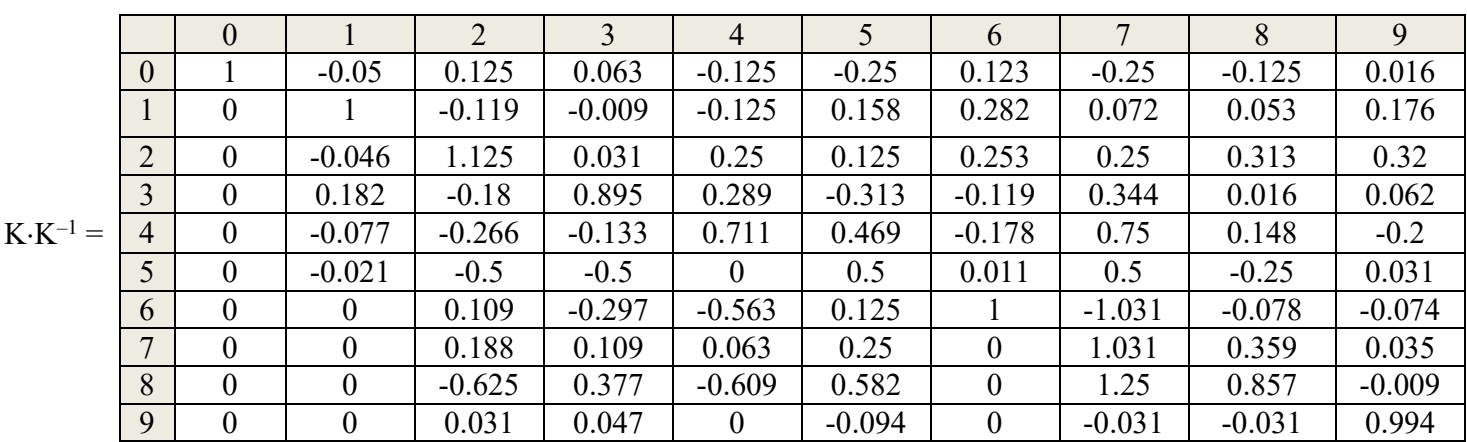




$\mathbf{K}:=\left(\begin{array}{ccccccccccccccccc}6.921 \cdot 10^{11} & -5.042 \cdot 10^{10} & 2.942 \cdot 10^{10} & -6.711 \cdot 10^{11} & 0 & -3.780 \cdot 10^{11} & 1.350 \cdot 10^{10} & -2.804 \cdot 10^{10} & 3.925 \cdot 10^{11} & 0 \\ -5.042 \cdot 10^{10} & 6.921 \cdot 10^{11} & 0 & -6.711 \cdot 10^{11} & 2.942 \cdot 10^{10} & 1.350 \cdot 10^{10} & 1.485 \cdot 10^{11} & 0 & -3.925 \cdot 10^{11} & 2.804 \cdot 10^{10} \\ 2.942 \cdot 10^{10} & 0 & 3.234 \cdot 10^{11} & -3.528 \cdot 10^{11} & 0 & 1.038 \cdot 10^{9} & 0 & 1.755 \cdot 10^{11} & -1.765 \cdot 10^{11} & 0 \\ -6.711 \cdot 10^{11} & -6.711 \cdot 10^{11} & -3.528 \cdot 10^{11} & 2.048 \cdot 10^{12} & -3.528 \cdot 10^{11} & 3.905 \cdot 10^{11} & -3.905 \cdot 10^{11} & -1.475 \cdot 10^{11} & -5.341 \cdot 10^{-5} & 1.475 \cdot 10^{11} \\ 0 & 2.924 \cdot 10^{10} & 0 & -3.528 \cdot 10^{11} & 3.234 \cdot 10^{11} & 0 & -1.038 \cdot 10^{9} & 0 & 1.765 \cdot 10^{11} & -1.755 \cdot 10^{11} \\ -3.780 \cdot 10^{11} & 1.350 \cdot 10^{10} & 1.038 \cdot 10^{9} & 3.905 \cdot 10^{11} & 0 & 1.040 \cdot 10^{12} & 3.246 \cdot 10^{11} & -3.846 \cdot 10^{11} & -9.801 \cdot 10^{11} & 0 \\ 1.350 \cdot 10^{10} & 1.485 \cdot 10^{11} & 0 & -3.905 \cdot 10^{11} & -1.038 \cdot 10^{9} & 3.246 \cdot 10^{11} & 1.040 \cdot 10^{12} & 0 & -9.801 \cdot 10^{11} & -3.864 \cdot 10^{11} \\ -2.804 \cdot 10^{10} & 0 & 1.755 \cdot 10^{11} & -1.475 \cdot 10^{11} & 0 & -3.846 \cdot 10^{11} & 0 & 4.554 \cdot 10^{11} & -7.085 \cdot 10^{10} & 0 \\ 3.925 \cdot 10^{11} & -3.925 \cdot 10^{11} & -1.765 \cdot 10^{11} & -5.341 \cdot 10^{-5} & 1.765 \cdot 10^{11} & -9.801 \cdot 10^{11} & -9.801 \cdot 10^{11} & -7.085 \cdot 10^{10} & 2.102 \cdot 10^{12} & -7.085 \cdot 10^{10} \\ 0 & 2.804 \cdot 10^{10} & 0 & 1.475 \cdot 10^{11} & -1.755 \cdot 10^{11} & 0 & -3.846 \cdot 10^{11} & 0 & -7.085 \cdot 10^{10} & 4.554 \cdot 10^{11}\end{array}\right)$.

Pис. 2. Матрица жесткости К, введенная в ручном режиме

Fig. 2. A manually inputed rigidity matrix $K$

В этом случае результат положительный. Значит, матрица жесткости, сформированная в ручном режиме, является верной. Подтверждение этому вычисление напряжений в конечных элементах I, II и III. Так как рассчитываемая конструкция (рис. 1) симметрична относительно вертикали, проходящей через вершину 4, и по геометрии, и по внешним силовым воздействиям, напряжения в конечных элементах I и III должны быть одинаковыми (касательные напряжения должны иметь разные знаки). Расчеты показывают, что этому условию удовлетворяет лишь решение, построенное на основе матрицы жесткости $\mathbf{K}$, сформированной путем ручного ввода значений ее компонент:

Определяем напряжения.

КЭ - I

$S I:=\left(\frac{1}{2}\right) \cdot(x 3 y 4+x 1 \cdot y 3+x 4 \cdot y 1-x 3 \cdot y 1-x 4 \cdot y 3-x 1 \cdot y 4)=1.26$; $\sigma x \mathrm{I}:=\left(\frac{1}{2 \cdot S I}\right) \cdot[A \cdot[(y 3-y 4) \cdot u 1+(y 4-y 1) \cdot u 3+(y 1-y 3) \cdot u 4]+$ $+C \cdot[(x 4-x 3) \cdot v 1+(x 1-x 4) \cdot v 3+(x 3-x 1) \cdot v 4]]=4.875 \times 10^{3} ;$ $\sigma y \mathrm{I}:=\left(\frac{1}{2 \cdot S I}\right) \cdot[C \cdot[(y 3-y 4) \cdot u 1+(y 4-y 1) \cdot u 3+(y 1-y 3) \cdot u 4]+$ $+A \cdot[(x 4-x 3) \cdot v 1+(x 1-x 4) \cdot v 3+(x 3-x 1) \cdot v 4]]=-1.105 \times 10^{4} ;$ $\tau x y \mathrm{I}:=\left(\frac{1}{2 \cdot S \mathrm{I}}\right) \cdot G \cdot[(x 4-x 3) \cdot u 1+(x 1-x 4) \cdot u 3+(x 3-x 1) \cdot u 4+$ $+(y 3-y 4) \cdot v 1+(y 4-y 1) \cdot v 3+(y 1-y 3) \cdot v 4]=2.73 \times 10^{3}$. KЭ - II $S I I:=\left(\frac{1}{2}\right) \cdot(x 4 y 2+x 1 \cdot y 4+x 2 \cdot y 1-x 4 \cdot y 1-x 2 \cdot y 4-x 1 \cdot y 2)=1.17$; $\sigma x \mathrm{II}:=\left(\frac{1}{2 \cdot S \mathrm{II}}\right) \cdot[A \cdot[(y 4-y 2) \cdot u 1+(y 2-y 1) \cdot u 4+(y 1-y 4) \cdot u 2]+$ $+C \cdot[(x 2-x 4) \cdot v 1+(x 1-x 2) \cdot v 4+(x 4-x 1) \cdot v 2]]=-5.093 \times 10^{3} ;$ $\sigma y \mathrm{II}:=\left(\frac{1}{2 \cdot S \mathrm{II}}\right) \cdot[C \cdot[(y 4-y 2) \cdot u 1+(y 2-y 1) \cdot u 4+(y 1-y 4) \cdot u 2]+$ $+A \cdot[(x 2-x 4) \cdot v 1+(x 1-x 2) \cdot v 4+(x 4-x 1) \cdot v 2]]=9.179 \times 10^{3} ;$ $\tau x y I I:=\left(\frac{1}{2 \cdot S I I}\right) \cdot G \cdot[(x 2-x 4) \cdot u 1+(x 1-x 2) \cdot u 4+(x 4-x 1) \cdot u 2+$ $+(y 4-y 2) \cdot v 1+(y 2-y 1) \cdot v 4+(y 1-y 4) \cdot v 2]=2.778$.
KЭ - III

$S \mathrm{III}:=\left(\frac{1}{2}\right) \cdot(x 4 y 5+x 2 \cdot y 4+x 5 \cdot y 2-x 4 \cdot y 2-x 5 \cdot y 4-x 2 \cdot y 5)=1.26$; $\sigma x \mathrm{III}:=\left(\frac{1}{2 \cdot \operatorname{SIII}}\right) \cdot[A \cdot[(y 4-y 5) \cdot u 2+(y 5-y 2) \cdot u 4+(y 2-y 4) \cdot u 5]+$ $+C \cdot[(x 5-x 4) \cdot v 2+(x 2-x 5) \cdot v 4+(x 4-x 2) \cdot v 5]]=4.879 \times 10^{3} ;$ $\sigma y \mathrm{III}:=\left(\frac{1}{2 \cdot S \mathrm{III}}\right) \cdot[C \cdot[(y 4-y 5) \cdot u 2+(y 5-y 2) \cdot u 4+(y 2-y 4) \cdot u 5]+$ $+A \cdot[(x 5-x 4) \cdot v 2+(x 2-x 5) \cdot v 4+(x 4-x 2) \cdot v 5]]=-1.104 \times 10^{4} ;$ $\tau x y \mathrm{III}:=\left(\frac{1}{2 \cdot S \mathrm{III}}\right) \cdot G \cdot[(x 5-x 4) \cdot u 2+(x 2-x 5) \cdot u 4+(x 4-x 2) \cdot u 5+$ $+(y 4-y 5) \cdot v 2+(y 5-y 2) \cdot v 4+(y 2-y 4) \cdot v 5]=-2.726 \times 10^{3}$.

Здесь SI, SII, SIII - площади конечных элементов $\left(\mathrm{м}^{2}\right) ; \sigma x, \sigma y, \tau x y-$ напряжения (Па), относящиеся к конечным элементам I, II и III.

\section{Заключение}

Для решения задач расчета строительных и машиностроительных конструкций методом конечных элементов возможностей математического пакета MathCAD явно недостаточно. Для задач учебного или иллюстративного характера, когда матрица жесткости имеет небольшую размерность и ее компоненты можно ввести в ручном режиме, возможностей пакета MathCAD вполне хватает и с его помощью можно получить решение задачи. В реальных расчетных задачах матрица жесткости имеет большой порядок (порядка тысяч, десятков и сотен тысяч), а потому для их решения следует использовать специализированные программные комплексы, например ЛИРА [10], SCAD [11], ANSYS [12] и другие.

Полученные результаты могут найти применение при решении задач расчета строительных и машиностроительных конструкций методом конечных элементов с использованием пакетов прикладных программ программно-математического обеспечения персональных компьютеров, в частности, математического пакета MathCAD. 


\section{Литература}

1. Бакушев С.В. Компьютерные технологии решения задач в архитектурно-строительном вузе // Смешанное и корпоративное обучение (СКО-2007): тр. Всерос. науч.-методич. симпоз. 2007. С. 110-113.

2. Рыков С.В., Кудрявцева И.В., Яворская А.Н., Камоцкий В.И. Использование пакета МathCAD при подготовке студентов экологических и экономических направлений // Науч. журн. НИУ ИТМО. Сер.: Экономика и экологический менеджмент. 2014. № 2. С. 16-24.

3. Половко A.M., Ганичев И.В. MathCAD для студента. СПб: БХВ-Петербург, 2010. 336 с.

4. Макаров Е.Г. Инженерные расчеты в MathCAD. СПб: Питер, 2005. 448 c.

5. Бакушев С.В. Сопротивление материалов. Выполнение расчетно-графических работ и решение задач с исполь- зованием математического пакета MathCAD. M., 2006.

6. Охорзин В.А. Прикладная математика в системе MathCAD. СПб: Лань, 2008. 352 с.

7. Зенкевич О., Чанг И. Метод конечных элементов в теории сооружений и в механике сплошных сред; [пер. с англ. А.П. Троицкого, С.В. Соловьева; под ред. Ю.К. Зарецкого]. М.: Недра, 1974. 240 с.

8. Розин Л.Ф. Метод конечных элементов в применении к упругим системам. М.: Стройиздат, 1977. 128 с.

9. Бате К., Вилсон Е. Численные методы анализа и метод конечных элементов. М.: Стройиздат, 1982. 448 с.

10. LiraLand. URL: http://www.liraland.ru (дата обращения: 16.11.2017).

11. SCAD Office. URL: http://www.scadsoft.com (дата обращения: 16.11.2017).

12. ANSYS Discovery Products. URL: http://www.ansys.com (дата обращения: 16.11.2017).

\title{
FINITE ELEMENT STRUCTURAL DESIGN IN THE MATHEMATICAL PACKAGE MathCAD
}

\author{
S.V.Bakushev ${ }^{1}$, Dr.Sc. (Engineering), Professor, bakuchsv@mail.ru
}

\section{${ }^{1}$ Penza State University of Architecture and Construction, German Titov St. 28, Penza, 440028, Russian Federation}

Abstract. The paper analyses mathematical package MathCAD potential when solving the problems of strength and deformation calculation of building and machine-building constructions using the finite element method. It is shown that MathCAD potentials are definitely not sufficient to solve such problems. It is due to the necessity to process quite a large amount of information.

For illustrative and study tasks, when a rigidity matrix has not big dimension and its components can be introduced manually, it is enough to use MathCAD. It can help to solve a problem. In this case, the rigidity matrix should be formed by manual introduction of its component data that were calculated beforehand in MathCAD. In real calculation tasks, a rigidity matrix has a large order (thousands, tens and hundreds of thousands). Therefore, there are specialized software packages to solve them, such as LIRA, SCAD, ANSYS, etc.

The paper considers a specific numerical example of calculating a compound profile plate under plain stress condition using the finite element method. The plate is separated into three finite elements. In this case, a rigidity matrix has the order equal to ten; the problem is solved only with manual introduction of its component data.

Keywords: finite element method, rigidity matrix, mathematical model MathCAD, personal computers.

\section{References}

1. Bakushev S.V. Computer technologies of problem solving at university of civil engineering and architecture. Smeshannoe i korporativnoe obuchenie (SKO-2007): tr. Vseros. nauch.-metodich. simpoz. [Proc. All-Russian Sci. and Methodol. Symp. Blended and Corporate Learning (SCO-2007)]. 2007, pp. 110-113 (in Russ.).

2. Rykov S.V., Kudryavtseva I.V., Yavorskaya A.N., Kamotsky V.I. Use mathcad at training of ecologists and economists students. Nauch. zhurn. NIU ITMO. Seriya "Ekonomika i ekologichesky menedzhment" [Scientific J. NRU ITMO. Series "Economics and Environmental Management"]. 2014, no. 2, pp. 16-24 (in Russ.).

3. Polovko A.M., Ganichev I.V. MathCAD dlya studenta [MathCAD for a Student]. St. Petersburg, BHV-Peterburg Publ., $2010,336 \mathrm{p}$.

4. Makarov E.G. Inzhenernye raschety v MathCAD [Engineering Calculations in MathCAD]. St. Petersburg, Peter Publ., $2005,448 \mathrm{p}$

5. Bakushev S.V. Soprotivlenie materialov. Vypolnenie raschetno-graficheskikh rabot i reshenie zadach s ispolzovaniem matematicheskogo paketa MathCAD [Material Resistance. Computational and Graphical Works and Problem Solving in a Mathematical Package MathCAD]. State registration number N 50200600581, GKTSIT, OFAP, Moscow, 2006.

6. Okhorzin V.A. Prikladnaya matematika v sisteme MathCAD [Applied Mathematics in the MathCAD System]. 2nd ed., St. Petersburg, Lan Publ., 2008, 352 p.

7. Zenkevich O., Chang I. The Method of Finite Elements in the Theory of Structures and in the Mechanics of Continuous Media. NY, 1967 (Russ. ed.: A.P. Troitsky, S.V. Solovev, Yu.K. Zaretsky (Eds.). Moscow, Nedra Publ., 1974, 240 p.

8. Rozin I.F. Metod konechnykh elementov v primenenii $k$ uprugim sistemam [Finite Element Method in Application to Elastic Systems]. Moscow, Stroiizdat Publ., 1977, 128 p.

9. Bathe K.J., Wilson E.L. Numerical Methods in Finite Element Analysis. Prentice-Hall Publ., Englewood Cliffs, NJ, 1976, 528 p. (Russ. ed.: Moscow, Stroiizdat Publ., 1982, 448 p.).

10. LiraLand. Available at: http://www.liraland.ru (accessed November 16, 2017)

11.SCAD Office. Available at: http://www.scadsoft.com (accessed November 16, 2017).

12. ANSYS Products. Available at: http://www.ansys.com (accessed November 16, 2017). 